\title{
Inter-Cell Interference Coordination in OFDMA Networks: A Novel Approach Based on Integer Programming
}

\author{
Mahmudur Rahman and Halim Yanikomeroglu \\ Department of Systems and Computer Engineering, Carleton University \\ 1125 Colonel By Drive, Ottawa, Ontario K1S 5B6, Canada \\ Email: $\{$ mmrahman,halim $\} @$ sce.carleton.ca
}

\begin{abstract}
We study a dynamic inter-cell interference coordination scheme formulated using a novel binary integer linear programming optimization technique. The scheme aims to maximize utility, where different utility measures have been defined to model varying levels of user fairness. The formulated problem is decomposed into a number of smaller sub-problems and solved iteratively to ease the computational complexity. We observe the cell-edge and sector throughput as well as user fairness and compare with a reference scheme, where interference coordination is not used. It is observed that the proposed scheme always outperforms the reference scheme in terms of cell-edge and sector throughput as well as in fairness.
\end{abstract}

\section{INTRODUCTION}

Orthogonal frequency division multiplexing (OFDM) based air-interface has been accepted in the beyond $3 \mathrm{G}$ to future $4 \mathrm{G}$ wireless systems, such as 3GPP Long Term Evolution (LTE) [1], LTE-Advanced (LTE-A) [2], worldwide interoperability for microwave access (WiMAX) [3], and world initiative new radio (WINNER) [4], due to its robustness to inter-symbol interference (ISI) resulting from frequency selective multi-path fading complimented by its suitability to flexible radio resource allocations. Multiuser OFDM or orthogonal frequency division multiple access (OFDMA) provides such flexibility of allocating and modulating each subcarrier adaptively to exploit frequencydomain diversity and improve achievable data rates on available frequency bandwidth.

The next generation wireless systems are required to employ dense reuse of spectrum in order to support envisioned high data rate wireless applications and services. As a result, intercell interference has been regarded as one of the key issues in designing these systems. To that end, studies on inter-cell interference coordination (ICIC) have recently been attracted researchers in academia and industry. In this paper, we formulate a dynamic ICIC scheme using integer linear programming (ILP) approach and solve it by decomposing the problem into a number of smaller sub-problems.

Interference avoidance using reuse partitioning has been first proposed in [5], where resources are reused with a clustering approach. This approach is effective for minimizing inter-cell interference, however, with significant resource loss due to partitioning. Early wireless networks supporting primarily voice services could cope with such resource loss. However, present and upcoming systems would have to support much higher data rates and static partition based approach is ineffective. A reuse of 1 or closer to 1 has recently been considered in next generation systems using fractional frequency reuse (FFR) techniques in [1], [3], [6]. A common example of FFR schemes achieve an effective frequency reuse between 1 and 3. Readers are referred to [6] for a detailed description of FFR schemes. These schemes, when used in static manner, can effectively reduce interference and boost cell-edge performance, however, only with considerable penalty to cell throughput [6]. Also, static interference coordination schemes require prior resource planning which makes it difficult for a network to have self organization capability and unsuitable for femto-cell [7] deployment. A dynamic interference coordination scheme is desirable not only to best exploit the mutual interference situation dynamically but also to offer applicability to future femto-cell environment.

In the literature, only a few studies can be found that focus on dynamic coordination [8]-[12]. The dynamic intercell interference coordination scheme in [8] uses a simplistic scenario, in which a reuse pattern is chosen from four predefined patterns with varying degrees of partitioning (e.g., reuse of 1 to reuse of 3). A dynamic FFR scheme has been studied in [9], where resources are dynamically partitioned into supergroup and regular-group and are allocated to cells and sectors, respectively. Although the scheme achieves higher system throughput, it shows degraded cell-edge performance compared to a static FFR scheme. A heuristic approach is used in the dynamic interference coordination scheme in [10]. Interference graph method is used in [11] and [12].

Interference coordination considering a detailed optimization framework has only been studied in a few studies. In this paper, we formulate interference coordination problem using integer linear programming (ILP) approach. This work is based on our previous work presented in [13]. However, there are a number of ways in which we have extended our previous work. While the emphasis in [13] was to achieve maximized throughput, this research focuses in achieving enhanced cell-edge performance. In this paper, we consider denser reuse (i.e., potential reuse of resources in each sector) instead of partitioning used in [13]. Also, we define different utility parameters to model different levels of fairness and aim to maximize utility.

The rest of this paper is organized as follows. Section II describes the system layout considered. The formulation of the algorithm is presented in Section III. The simulation and system parameters are described in Section IV. We discuss results in Section $\mathrm{V}$ followed by conclusions in Section VI. 


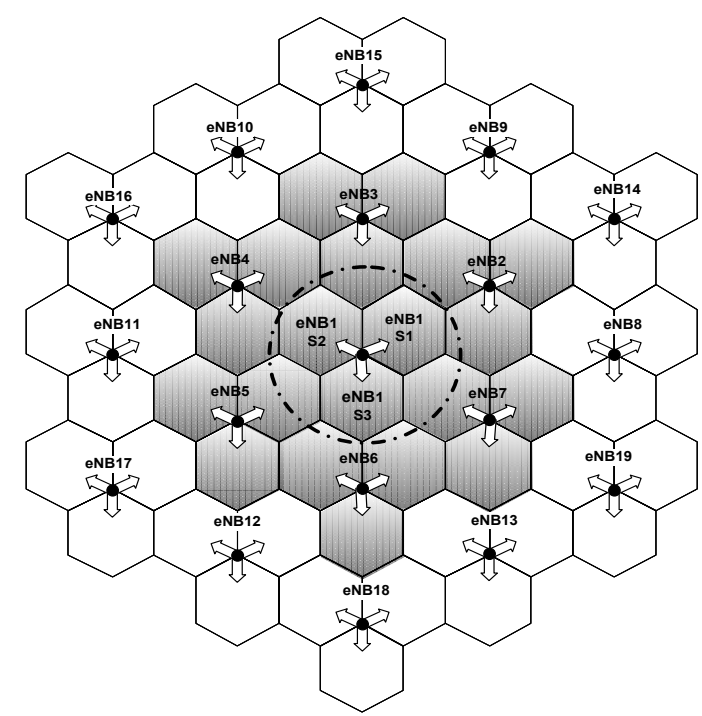

Fig. 1. Investigated network layout.

\section{SYSTEM MODEL}

A network layout consisting of 19 cell sites each with 3 hexagonal sectors, as shown in Fig. 1, is considered in this study. Directional transmit antennas with $120^{\circ}$ beamwidth (3 $\mathrm{dB}$ beamwidth of $70^{\circ}$ ) at each sector and omni-directional receive antennas at user equipments (UEs) are considered. The antenna gain pattern of the transmit antenna is described in Section IV. It is assumed that the system uses cell-specific orthogonal reference signals [1] and UEs know the reference signals of neighboring first-tier sectors; therefore, UEs can estimate interference from the first-tier sectors separately. For a downlink transmission to a UE in any sector, one of its first-tier sectors is likely to be the most dominant interferer. Take Fig. 1 as an example, a cell-edge UE in sector 1 of evolved NodeB 1 (eNB1) is likely to receive the most dominant interference from sector 2 or 3 of eNB1 (depending on the UE location), or from sector 2 or 3 of eNB2, or from sector 3 of eNB3, or from sector 2 of eNB7 due to their relative locations and antenna directivity.

In addition to its higher path-loss, a cell-edge UE receives significant interference from the nearby sectors. As a consequence, these UEs are expected to see more poor-quality resource blocks (RBs) having low signal-to-interference-plus-noise ratios (SINRs). A RB is defined as the minimum granularity of timefrequency resource unit for scheduling [1]. An optimal or a sub-optimal allocation scheme with an objective to maximize network throughput will tend to overlook such disadvantaged UEs due to their poor contribution to the total throughput. Therefore, it is crucial to avoid interference on such UEs in order to guarantee their minimum required rates.

Maximization of user rates is only meaningful when user traffic is insensitive to delay, i.e., elastic traffic [14]. Therefore, we introduce a utility measure as a function of rate and user service status to make our algorithm suitable for delay sensitive services. The algorithm can reside at any centralized or semicentralized logical entity to run centrally or in a semi-distributed manner.
TABLE I

LIST OF SYMBOLS USED

$k \quad$ index of the second dominant interfering sector

$u \quad$ UE index

$b \quad$ RB index

$M \quad$ number of UEs per sector

$N \quad$ number of available RBs per sector

$P_{b} \quad$ power per RB

$P_{T N} \quad$ average thermal noise power

$\mathcal{H}^{(u, b)} \quad$ channel gain seen by UE $u$ on RB $b$

$\gamma^{(u, b)} \quad$ SINR experienced by UE $u$ on RB $b$

$r^{(u, b)} \quad$ achievable rate seen by UE $u$ on RB $b$

$d^{(u)} \quad$ UE demand factor

$I^{(b)} \quad$ indicator to show whether RB $b$ is restricted or not

$R^{(u)} \quad$ time average throughput achieved by UE $u$

$\bar{R} \quad$ average throughput across all UEs

For convenience, we provide a list of the symbols used in this paper in Table I. In our scheme, we first determine two most dominant interferers among the first-tier interfering sectors for each user equipment (UE). A UE determines its strongest interferer(s) among the first-tier sectors from cellspecific orthogonal reference signals. The mean interference power (averaged over short-term variation), seen by UE $u$ at sector $i$, can be given by the following expression:

$$
\mathcal{I}_{i, \mathbf{f}}^{(u)}=P_{b} \cdot \mathcal{H}_{i, \mathbf{f}}^{(u)}
$$

where $\mathbf{f}$ is the set of first-tier dominant interferer sectors, $P_{b}$ is the transmit power, and $\mathcal{H}_{\mathbf{f}}$ is the path-gain including pathloss, shadowing, and antenna gains. We sort $\mathcal{I}_{i, \mathbf{f}}^{(u)}$ according to descending powers of received interference. Let us denote $j$ and $k$ as the indices of the first and the second interfering sectors to UE $u$ in sector $i$. An interferer group for UE $u$ is constructed with the indices of these sectors as

$$
\mathcal{G}_{i}^{(u)}=\{j, k\} .
$$

We construct an interferer group for each UE in each sector following above method. These groups remain unchanged as long as the user locations do not change. It is possible that the group can be determined dynamically based on short-term channel variation resulting from multi-path fading; however, this will introduce added complexity due to an additional dimension needed to account for channel variability across RBs. Once the groups are determined, we calculate achievable rates UEs foresee for different interferer restriction possibilities such as when none, one, and two interferer(s) is(are) restricted. For a RB $b$, we express these rates as $r_{i \mid\{\}}^{(u, b)}, r_{i \mid j}^{(u, b)}, r_{i \mid k}^{(u, b)}$, and $r_{i \mid(j, k)}^{(u, b)}$ denoting no restriction, $j$ restricted, $k$ restricted, and $(j, k)$ restricted, respectively. The rates are calculated from the following SINR expression:

$$
\gamma_{i}^{(u, b)}=\frac{P_{b} \mathcal{H}_{i, i}^{(u, b)}}{P_{b} \sum_{\substack{\Psi \neq i \\ \Psi \notin \mathcal{G}_{i}^{(u)}}} \mathcal{H}_{i, \Psi}^{(u, b)}+P_{b} \sum_{x \in \mathcal{G}_{i}^{(u)}} \mathcal{H}_{i, x}^{(u, b)} \cdot I_{x}^{(b)}+P_{T N}}
$$


where $P_{b}$ is the transmit power per RB. The channel gains for the desired and interferer links are $\mathcal{H}_{i, i}^{(u, b)}$ and $\mathcal{H}_{i, \Psi}^{(u, b)}$, respectively. The parameter $\mathcal{H}$ includes path-loss, fading, shadowing, and antenna gains. The parameter $P_{T N}$ denotes average thermal noise power experienced within the RB.

Let us denote $\Pi$ as the set of transmission restriction possibilities of in-group dominant interfering sectors, i.e., $\{\{\},\{j\},\{k\},\{j, k\}\}$. Then, $\gamma_{i \mid \Pi}^{(u, b)}=\gamma_{i}^{(u, b)}$ with $I_{x}^{(b)}=0$ for $x \in \Pi$ and $I_{x}^{(b)}=1$ for $x \notin \Pi$. The decision of which RB is to be restricted in which sector is determined by the solution of the optimization problem as described in the subsequent section.

We consider 4 different scenarios with 4 types of utilities $(U)$ for various degrees of emphasis on user scheduling and fairness: 1) $U_{i}^{(u, b)}=r_{i}^{(u, b)}$, 2) $\left.U_{i}^{(u, b)}=r_{i}^{(u, b)} d_{i}^{(u)}, 3\right)$ $U_{i}^{(u, b)}=r_{i}^{(u, b)}\left[d_{i}^{(u)}\right]^{2}$, and 4) $U_{i}^{(u, b)}=\left[r_{i}^{(u, b)}\right]^{2} d_{i}^{(u)}$. Here, we define UE demand factor $d_{i}^{(u)}$ as follows: $d_{i}^{(u)}=\bar{R}_{i} / R_{i}^{(u)}$, where $R_{i}^{(u)}$ is the average throughput of UE $u$ over a certain past time-window (10 RBs long time duration is considered in simulations), and $\bar{R}_{i}$ is the average throughput across all UEs and is given by $\bar{R}_{i}=\left(\sum_{u=1}^{M} R_{i}^{(u)}\right) / M$, and $M$ is the number of UEs per sector. The first scenario aims to maximize rate only, while the others consider fairness with varying degree by incorporating UE demand in the utility measure. A UE near the cell-edge will likely have a higher $d_{i}^{(u)}$. Therefore, the utilities incorporating $d_{i}^{(u)}$ provide advantages to rate deprived UEs to boost their performance.

\section{Problem Formulation}

We formulate the utility maximization problem as follows:

maximize

$$
\sum_{i} \sum_{\Pi}\left[\sum_{u=1}^{M} \sum_{r=1}^{N} U_{i \mid \Pi}^{(u, b)} \rho_{i \mid \Pi}^{(u, b)}\right],
$$

subject to

$$
\begin{gathered}
\rho_{i \mid \Pi}^{(u, b)} \in\{0,1\} ; \forall\{u, b\}, \\
I_{i}^{(b)}=\sum_{\Pi} \sum_{u=1}^{M} \rho_{i \mid \Pi}^{(u, b)}= \begin{cases}0 ; & \text { RB } b \text { is restricted in } i \\
1 ; & \text { otherwise. }\end{cases}
\end{gathered}
$$

where constraints in (5) indicate that the problem is binary integer type and those in (6) imply that if RB $b$ is not restricted in $i$, it can be assigned to only one UE.

As described earlier, $\Pi_{i}^{(u, b)}$ defines four possible choices of dominant interferer transmissions on RB $b$. For example, if the first and the second dominant interferers to $i$ are $j$ and $k$, respectively, then the possibilities are as follows: 1) $j$ and $k$ are not restricted to use RB $b$ (i.e., $I_{j}^{(b)}=1, I_{k}^{(b)}=1$ ) and sector $i$ achieved rate is $r_{i \mid\{\}}^{(u, b)}$, 2) $j$ is restricted (i.e., $I_{j}^{(b)}=0, I_{k}^{(b)}=1$ ) and rate in sector $i$ is $r_{i \mid j}^{(u, b)}$, 3) $k$ is restricted (i.e., $I_{j}^{(b)}=1$, $\left.I_{k}^{(b)}=0\right)$ and rate in sector $i$ is $r_{i \mid k}^{(u, b)}$, and 4) both $j$ and $k$ are restricted (i.e., $I_{j}^{(b)}=0, I_{k}^{(b)}=0$ ) and rate in sector $i$ is $r_{i \mid\{j, k\}}^{(u, b)}$. Clearly, sector $j$ and $k$ will have their restrictions to their dominant interferer sectors and this inter-relation propagates in the network. These restrictions and concurrent transmissions possibilities are modeled by the following set of constraints:

$$
\begin{aligned}
\rho_{i \mid j}^{(u, b)}+I_{j}^{(b)} & =\{0,1\}, \\
\rho_{i \mid k}^{(u, b)}+I_{k}^{(b)} & =\{0,1\}, \\
\rho_{i \mid\{j, k\}}^{(u, b)}+I_{j}^{(b)} & =\{0,1\}, \\
\rho_{i \mid\{j, k\}}^{(u, b)}+I_{k}^{(b)} & =\{0,1\} .
\end{aligned}
$$

The complexity of the integer programming increases with the number of variables and constraints. Solving this problem network-wide as given by (4) through (7) with all available RBs at a time is computationally prohibitive. Therefore, we break down the problem into a number of smaller sub-problems and solve each of the sub-problems at a time. Each subproblem only takes a sub-set of available RBs (for instance, $\kappa \in\{1,2, \cdots, N\}$; we assume the size of $\kappa$ as $10 \mathrm{RBs}$ in simulations) and assigns among UEs following (4) through (7). Also, in order to restrict excessive resource assignment to a UE, we introduce the following constraint so that in each iteration of sub-problems a UE is assigned a maximum of 2 RBs:

$$
\sum_{\Pi} \sum_{b} \rho_{i \mid \Pi}^{(u, b)} \leq 2 ; \quad \forall\{i, u\} .
$$

The pseudo codes of the algorithm is given in Table II, where each sub-problem is solved iteratively until all chunks are assigned in all sectors following (4) to (8).

\section{System And Simulation Parameters}

We have considered a total of 19 cell sites (i.e., 57 hexagonal sectors) in the simulations, as shown in Fig. 1. The intersite distance is assumed to be $500 \mathrm{~m}$. The UEs are randomly distributed in the centre 21 shaded sectors on a circular disk with a minimum and maximum radius in each sector. The closein distance is assumed to be $35 \mathrm{~m}$. We observe performance statistics from the 3 central sectors of eNB1 as marked by the circle in Fig. 1.

The large-scale path-loss including penetration loss and shadowing can be expressed as follows [15],

$$
L_{D}=128.1+10 n \log _{10}(D)+L_{P}+X_{\sigma},
$$

where $L_{D}$ is the distance dependent path-loss in $\mathrm{dB}, D$ is the separation distance in $\mathrm{km}$ between the transmitter and the receiver of interest, $L_{P}$ is the penetration loss in $\mathrm{dB}$, and $X_{\sigma}$ is a Gaussian random variable with $\sigma \mathrm{dB}$ standard deviation accounting for shadowing.

TABLE II

Pseudo Codes of the Algorithm

Initialize the list of unassigned $\mathrm{RBs} \mathcal{L}=\{1,2, \ldots, N\}$

while $\mathcal{L} \neq\{\}$ do

Take a set of RBs, $\kappa$, from unassigned set $\mathcal{L} ; \kappa \in \mathcal{L}$.

Solve optimization problem given by (4) to (8).

Update $R, \bar{R}$, and $d$ for all UEs in all sectors.

Remove $\kappa$ RBs from $\mathcal{L}$.

end while 
We assume that eNB transmit and UE receive antennas are $120^{\circ}$ directional and omni-directional, respectively. The horizontal antenna pattern of the sector antenna is considered as follows [15]:

$$
A(\theta)=-\min \left[12\left(\frac{\theta}{\theta_{3 d B}}\right)^{2}, 20\right],
$$

where $\theta$ varies from $-180^{\circ}$ to $180^{\circ}$ and $\theta_{3 d B}$ is $70^{\circ}$. We have used a single-in-single-out (SISO) antenna configuration.

The extended spatial channel model (SCME) with 6-taps has been considered to generate time-frequency correlated fading samples using the power delay profile defined in [16]. The Doppler frequency has been calculated with UE speed of 30 $\mathrm{km} / \mathrm{hr}$. We consider independent lognormal shadow fading with a standard deviation of $8 \mathrm{~dB}$. The average thermal noise is calculated with a noise figure of $7 \mathrm{~dB}$.

$\mathrm{A} \mathrm{RB}$ is made up of 12 consecutive subcarriers each of 15 $\mathrm{KHz}$ and it spans over 7 OFDM symbols time duration [1]; therefore, a RB has 84 OFDM symbols. We consider $10 \mathrm{MHz}$ system bandwidth (in $2.0 \mathrm{GHz}$ band) which constitutes a total of 50 RBs. Resources are reused in every sector, and thus each sector transmitter is a potential interferer to UEs in other sectors. We consider that downlink reference and control signals occupy 4 and 3 OFDM symbols, respectively, leaving 77 OFDM symbols per RB for data traffic.

We use a continuous rate adaptive modulation and coding scheme derived from an attenuated and truncated form of Shannon bound that matches with link level performance curves for modulation levels of quadrature phase shift keying (QPSK), 16and 64-quadrature amplitude modulation (QAM), and coding rates ranging from $1 / 8$ to $4 / 5$ [17]. The following expression shows the relation between SINR and corresponding spectral efficiency:

$$
\eta= \begin{cases}0 ; & \gamma<\gamma_{\min } \\ \alpha S(y) ; & \gamma_{\min }<\gamma<\gamma_{\max } \\ \eta_{\max } ; & \gamma>\gamma_{\max }\end{cases}
$$

where $\gamma$ is the average SINR seen on a RB and $\eta$ is the spectral efficiency in bps/Hz. The parameter $\alpha$ is the attenuation factor with regard to the Shannon bound, $S(\gamma)=\log _{2}(1+\gamma)$. The value of $\alpha$ is 0.75 for the modulation and coding schemes used. The maximum spectral efficiency, $\eta_{\max }$, is $4.8 \mathrm{bps} / \mathrm{Hz}$ which is achieved at maximum SINR, $\gamma_{\max }$, of $19.2 \mathrm{~dB}$. The minimum SINR is $\gamma_{\min }(-6.5 \mathrm{~dB}$ in simulations) below which the $\mathrm{RB}$ is unusable.

We have used MATLAB along with optimization solvers YALMIP [18] and TOMLAB/CPLEX [19]. For convenience, we provide Table III, where system and simulation parameters are summarized.

\section{Simulation Results}

For each utility scenario, we consider a corresponding reference scheme which also maximizes the same utility parameter with the difference that the reference scheme does not have the capability of interference coordination. Therefore, the gain observed in the proposed scheme is entirely due to inter-cell
TABLE III

System AND Simulation PARAMETERS
Cellular layout

Inter-site distance

Carrier frequency and Bandwidth

Path-loss exponent

Lognormal shadowing

Shadowing standard deviation

UE speeds

Penetration loss

Antenna configuration

eNB antenna gain

UE noise figure

AMC modes

Channel model

Total sector TX power

UE close-in distance

Traffic model
UE antenna gain
Hexagonal grid, 19 cell sites, 3 sectors per site $500 \mathrm{~m}$

$2.0 \mathrm{GHz}$ and $10 \mathrm{MHz}$ (50 RBs) 3.76

Independent among links

$8 \mathrm{~dB}$

$30 \mathrm{~km} / \mathrm{hr}$

$10 \mathrm{~dB}$

Single-input single-output

$14 \mathrm{dBi}$

$0 \mathrm{dBi}$

$7 \mathrm{~dB}$

Attenuated Shannon bound for QPSK, 16- and 64-QAM with varying rates

6-Tap SCME

$46 \mathrm{dBm}$

$35 \mathrm{~m}$

Full buffer interference coordination. The cell-edge throughput (measured as the $5^{\text {th }}$-percentile point of the CDF of time-averaged UE throughput), average sector throughput (averaged over sectors), the $95^{\text {th }}$-percentile UE throughput, and Jain's fairness index [20] have been considered to compare the performance of the simulated schemes for different utility scenarios as discussed in Section II.

In each of the central 21 sectors, $10 \mathrm{UEs} /$ sector are placed randomly for each simulation drop. Interferers are coordinated in these sectors only; the remaining sectors act as interference contributors only. Each drop is simulated for $30 \mathrm{RBs}$ time duration and a total of 70 drops is considered for each utility scenario. Statistics are collected from the central 3 sectors belonging to eNB1. User demands $d_{i}$ is calculated from the past throughput achieved over $10 \mathrm{RBs}$ time duration.

The CDF of average UE throughput is shown in Fig. 2. In the inset of the figure, the tail of the CDF is zoomed for the clarity to observe the $5^{\text {th }}$-percentile point. The observed performance parameter from this figure is also summarized in Table IV. We observe that $U=r$ scenario shows minimal benefit to the cell-edge UEs as the scheme aims to maximize the rate. However, it achieves the most sector throughput. The $U=r d^{2}$ scenario with ICIC shows the most gain in terms of cell-edge throughput, which is better that $U=r d$ scenario. The $U=r d^{2}$ achieves the least sector and the $95^{\text {th }}$-percentile UE throughput showing clear trade-off between cell-edge and sector throughput. The scenario $U=r^{2} d$ achieves better cell-edge throughput compared to that in $U=r$ scenario (with comparable sector throughput) as the utility measure includes the UE demand. Average sector throughput in all ICIC schemes has always been higher compared to that in the corresponding reference schemes.

We compare achieved fairness index using Jain's fairness index in Fig. 3. It is observed that schemes with ICIC for all utility scenarios show better fairness compared to those where ICIC is not used. The $U=r d^{2}$ scenario with ICIC exhibits the 


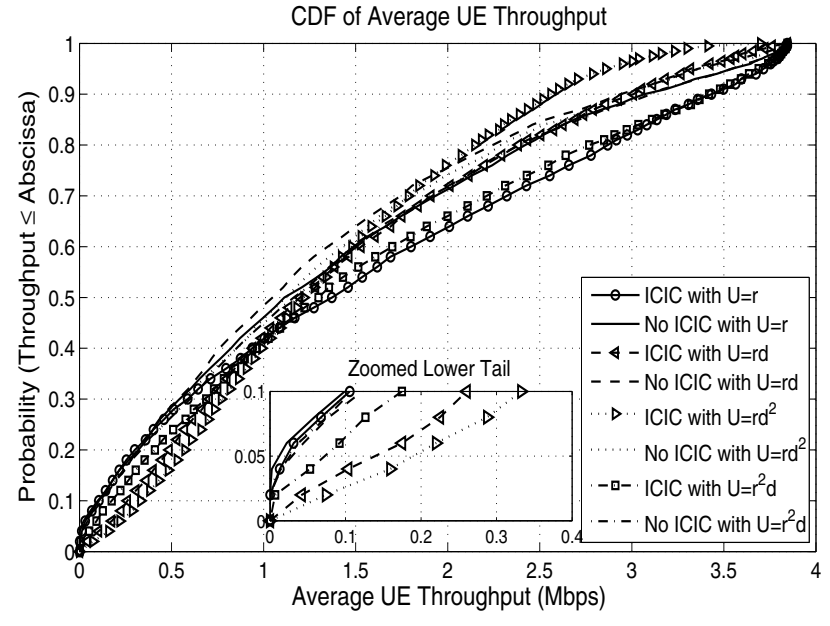

Fig. 2. CDF of average UE throughput for different utility scenarios.

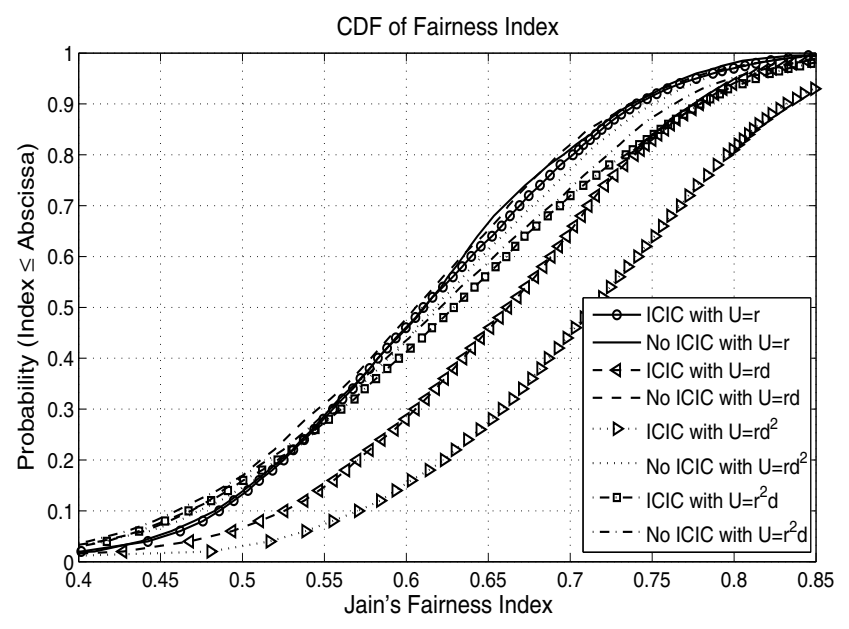

Fig. 3. CDF of Jain's fairness index.

highest fairness index.

\section{CONCLUSION}

In this paper, we have studied a dynamic inter-cell interference coordination (ICIC) scheme for downlink OFDMA system. Different utility parameters have been defined to model various levels of user fairness. A novel integer linear programming formulation has been devised to formulate ICIC that maximizes the defined utility parameters. The problem is decomposed into

TABLE IV

Comparison of Cell-Edge ( $5^{\text {th }}$ Percentile), $95^{\text {th }}$ Percentile, And AVERAGE SECTOR ThroughPUT

\begin{tabular}{c|c|c|c}
\hline $\begin{array}{c}\text { Utility } \\
\text { Scenario }\end{array}$ & $\begin{array}{c}5^{\text {th }} \text {-Perc. } \\
(\mathrm{kbps})\end{array}$ & $\begin{array}{c}95^{\text {th }} \text {-Perc. } \\
(\mathrm{Mbps})\end{array}$ & $\begin{array}{c}\text { Sector TP } \\
(\mathrm{Mbps})\end{array}$ \\
\hline \hline$U=r$ & $23.1(12.0)$ & $3.72(3.54)$ & $15.63(13.75)$ \\
$U=r d$ & $139.2(28.4)$ & $3.32(3.36)$ & $14.28(12.89)$ \\
$U=r d^{2}$ & $186.4(24.5)$ & $2.86(3.37)$ & $13.50(13.33)$ \\
$U=r^{2} d$ & $74.4(25.0)$ & $3.69(3.54)$ & $15.54(13.86)$
\end{tabular}

(Performance figures of the corresponding reference scheme (without ICIC) are shown in parenthesis.) a number of smaller sub-problems and solved iteratively with a reduced level of computational complexity.

It is observed that schemes employing ICIC always outperform corresponding the reference schemes without coordination, with regard to cell-edge performance, sector throughput, and fairness. In particular, the utility scenario $U=r d^{2}$ with ICIC achieves 7.5 times higher cell-edge throughput, slightly higher sector throughput, and substantially better fairness compared to those in the corresponding reference scheme without ICIC.

\section{REFERENCES}

[1] E-UTRA and E-UTRAN Overall Description; Stage 2 (Release 8), 3GPP Technical Specification TS 36.300 V9.1.0, Sep. 2009. [Online]. Available: http://www.3gpp.org.

[2] Further Advancements for E-UTRA Physical Layer Aspects (Release 9), 3GPP Technical Specification TR 36.814 V0.4.1, Feb. 2009. [Online]. Available: http://www.3gpp.org.

[3] "Mobile WiMAX- part I: A technical overview and performance evaluation," White Paper, WiMAX Forum, Aug. 2006.

[4] Initial Report on Advanced Multiple Antenna Systems, WINNER+ Deliverable D1.4, Jan. 2009. [Online]. Available: http://projects.celticinitiative.org/winner+.

[5] V. MacDonald, "The cellular concept," Bell System Technical Journal, vol. 58, pp. 15-41, Jan. 1979.

[6] Interference Avoidance Concepts, WINNER II Deliverable D4.7.2, Jun. 2007. [Online]. Available: http://www.ist-winner.org.

[7] V. Chandrasekhar, J. Andrews, and A. Gatherer, "Femtocell networks: a survey," IEEE Communications Magazine, pp. 59-67, Sep. 2008.

[8] Further Discussion on Adaptive Fractional Frequency Reuse, 3GPP Project Document R1-072762, Jun. 2007. [Online]. Available: http://www.3gpp.org.

[9] S. H. Ali and V. C. M. Leung, "Dynamic frequency allocation in fractional frequency reused ofdma networks," IEEE Transactions on Wireless Communications, vol. 8, pp. 4286-4295, Aug. 2009.

[10] M. Rahman, H. Yanikomeroglu, and W. Wong, "Interference avoidance with dynamic inter-cell coordination for downlink LTE system," in Proc. IEEE Wireless Communications and Networking Conference (WCNC2009), Apr. 2009

[11] M. C. Necker, "Scheduling constraints and interference graph properties for graph-based interference coordination in cellular OFDMA networks," Springer Journal on Mobile Networks and Applications, vol. 14, pp. 539550, Jan. 2009.

[12] R. Y. Chang, Z. Tao, J. Zhang, and C.-C. J. Kuo, "Multicell OFDMA downlink resource allocation using a graphic framework," IEEE Transaction on Vehicular Technology, vol. 58, pp. 3494-3507, Sep. 2009.

[13] M. Rahman and H. Yanikomeroglu, "Multicell downlink OFDM subchannel allocations using dynamic intercell coordination," in Proc. IEEE Global Telecommunications Conference (GLOBECOM2007), Nov. 2007, pp. 5220-5225.

[14] F. P. Kelly, A. K. Maulloo, and D. K. H. Tan, "Rate control for communication networks: shadow prices, proportional fairness and stability," Journal of the Operational Research Society, vol. 49, pp. 237-252, Mar. 1998.

[15] Physical Layer Aspects for Evolved Universal Terrestrial Radio Access (UTRA) (Release 7), 3GPP Technical Report TR 25.814 V7.1.0, Sep. 2006. [Online]. Available: http://www.3gpp.org.

[16] D. S. Baum, J. Salo, M. Milojevic, P. Kyösti, and J. Hansen, "An interim channel model for beyond-3G systems," in Proc. IEEE Vehicular Technonogy Conference (VTC2005-Spring), May 2005, pp. 3132-3136.

[17] E-UTRA Radio Frequency (RF) System Scenarios (Release 8), 3GPP Technical Report TR 36.942 V1.2.0, Jun. 2007. [Online]. Available: http://www.3gpp.org.

[18] J. Löfberg, "YALMIP: a toolbox for modeling and optimization in MATLAB," in Proc. IEEE Computer-Aided Control System Design (CACSD) Conference, Taipei, Taiwan, 2004.

[19] TOMLAB Optimization Tool. (2009) Version 7.2. [Online]. Available: http://www.tomopt.com

[20] R. Jain, D. M. Chiu, and W. Hawe, "A quantitative measure of fairness and discrimination for resource allocation in shared systems," DEC, Research Report TR-301, 1984. 